\title{
ON A NOTE BY H. SCHWERDTFEGER
}

\author{
Peter Scherk \\ (received May 13, 1958)
}

Let $x, y$ denote real column vectors with $n$ elements. Let $A$ be a regular symmetric real $n \times n$ matrix. Dashes indicate transposition.

If $x$ is fixed, $x^{\prime} A x>0$, the discriminant of $A$ at $x$ is the quadratic form

$y^{\prime S y}$ where $S=S(x)=x^{\prime} A x \cdot A-A x x^{\prime} A$.

In Can. Math. Bull. 1, pp.175-179,Dr. Schwerdtfeger proved the equivalence of the following properties of $\mathrm{A}$ :

(i) $\mathrm{A}$ is of the congruence type $[+,-, \ldots,-]$.

(ii) $y^{\prime} \mathrm{Sy} \leqslant 0$ for all $y$, equality holding if and only if $y$ is a multiple of $x$. His note is of particular interest because he also discusses the eigen-values of $S$. If only the quoted result is aimed at, the following procedure may be shorter.

Following Dr. Schwerdtfeger, we transform $A$ into its congruence normal form $\mathrm{J}$. If the image of the fixed vector $x$ is again denoted by $x$, we have

$$
x^{\prime} J x=\sum_{1}^{p} x_{k}^{2}-\sum_{p+1}^{n} x_{k}^{2}>0 ;
$$

here $1 \leqslant p \leqslant n$.

Any vector y permits a unique decomposition

(2) $y=z+\lambda x \quad$ where $x^{\prime} J z=0$.

On account of $S x=0$, this leads to $S y=S z+\lambda S x=S z$ and

$$
y^{\prime} S y=z^{\prime S z}=x^{\prime} J x \cdot z^{\prime} J z
$$

If $A$ is positive definite, we have $p=n$ and $z^{\prime} \mathrm{Jz}>0$. By (1) and (3), S will be non-negative definite.

Now let $p=1$. Then by (1) and (2)

Can. Math. Bull., vol. 1, no. 3, Sept. 1958 


$$
x_{1}^{2}>\sum_{2}^{n} x_{k}^{2} \quad, \quad x_{1} z_{1}=\sum_{2}^{n} x_{k} z_{k}
$$

Hence bySchwarz's inequality

$$
\begin{aligned}
& x_{1}^{2} \cdot z^{\prime} \cdot J z=x_{1}^{2}\left(z_{1}^{2}-\sum_{2}^{n} z_{k}^{2}\right)=\left(\sum_{2}^{n} x_{k} z_{k}\right)^{2}-x_{1}^{2} \cdot \sum_{2}^{n} z_{k}^{2} \\
\leq & \left(\begin{array}{ll}
\sum_{2} & x_{k} z_{k}
\end{array}\right)^{2}-\left(\sum_{2}^{k} x_{k}{ }^{2}\right) \cdot\left(\sum_{2}^{k} z_{k}^{2}\right) \leq 0
\end{aligned}
$$

or $z^{\prime} J z \leqslant 0$, equality holding if and only if $z=0$. Thus (3) shows that (i) implies (ii).

$$
\text { If } \mathrm{p}=\mathrm{n}-1,(1) \text { and (2) read }
$$

(4) $x_{n}^{2}-\sum_{1}^{n-1} x_{k}^{2}<0, x_{n} z_{n}=\sum_{1}^{n-1} x_{k} z_{k}$

Any non-trivial solution $z$ of $\sum_{1}^{n-1} x_{k} z_{k}=0, z_{n}=0$ will satisfy (4) and

$$
z^{\prime} \mathrm{Jz}=\sum_{1}^{\mathrm{n}-1} z_{k}^{2}-z_{\mathrm{n}}^{2}>0 \text {. }
$$

On the other hand,

$$
z^{\prime}=\left(x_{n} x_{1}, \ldots, x_{n} x_{n-1}, \sum_{1}^{n-1} x_{k}^{2}\right)
$$

satisfies (4) and

$$
z^{\prime} J z=x_{n}^{2} \sum_{1}^{n-1} x_{k}^{2}-\left(\sum_{1}^{n-1} x_{k}^{2}\right)^{2}=\sum_{1}^{n-1} x_{k}^{2}\left(x_{n}^{2}-\sum_{1}^{n-1} x_{k}^{2}\right)<0,
$$

Finally, let $1<p<n-1$. Then there are numbers

$$
t_{1}, \ldots, t_{p} ; t_{p+1}, \ldots, t_{n}
$$

satisfying

$$
\begin{gathered}
\sum_{1}^{p} x_{i} t_{i}=0, \sum_{1}^{p} t_{i}^{2}>0 ; \sum_{p+1}^{n} x_{i} t_{i}=0, \sum_{p+1}^{n} t_{i}^{2}>0 . \\
z^{\prime}=\left(t_{1}, \ldots, t_{p}, \rho t_{p+1}, \ldots, \rho t_{n}\right) .
\end{gathered}
$$

Thus $x^{\prime} J z=0:$ The function

$$
f(\rho)=z^{\prime} J z=\sum_{1}^{p} t_{i}{ }^{2}-\rho^{2} \sum_{p+1}^{n} t_{i}{ }^{2}
$$

is positive for $\rho=0$, negative for large $\rho$. Thus (ii) does not hold if (i) if not satisfied.

University of Saskatchewan 